\title{
Computation of Pólya Polynomials of Primitive Permutation Groups
}

\section{By Rudolf Land}

\begin{abstract}
An almost complete list of Pólya polynomials of all primitive permutation groups up to degree 20 has been computed.

The number-theoretical interpretation of Pólya polynomials and van der Waerden's test make this a good tool to find safe conjectures for determining the group of an equation.

This work was encouraged and supported by Professor W. Jehne, Universität zu Köln.
\end{abstract}

1. Definitions. Let $G \leqslant \widetilde{S}_{n}$ be a permutation group of degree $n \in \mathbf{N}$. Then, any permutation $\sigma \in G$ has a unique factorization into a product of cycles. If this factorization consists of $t_{1}$ 1-cycles, $t_{2}$ 2-cycles, ... and $t_{n} n$-cycles, we call $\mathbf{t}:=\left(t_{1}, \ldots, t_{n}\right) \in \mathbf{N}_{0}^{n}$ the type of the permutation $\sigma$, which will be denoted by $\mathbf{t}(\sigma)=\left(t_{1}(\sigma), \ldots, t_{n}(\sigma)\right)$. Obviously we have

$$
\sum_{i=1}^{n} i t_{i}=\sum_{i=1}^{n} i t_{i}(\sigma)=n .
$$

For the permutation group $G$, we define the Polya polynomial as

$$
P_{G}\left(z_{1}, \ldots, z_{n}\right):=\frac{1}{\sharp G} \sum_{\sigma \in G} \mathbf{z}^{\mathbf{t}(\sigma)} \in \mathbf{Q}\left[z_{1}, \ldots, z_{n}\right],
$$

where

$$
\mathbf{z}:=\left(z_{1}, \ldots, z_{n}\right), \quad \mathbf{t}(\sigma)=\text { type of } \sigma, \quad \mathbf{z}^{\mathrm{t}}:=\prod_{i=1}^{n} z_{i}^{t_{i}}
$$

For $\mathbf{t} \in \mathbf{N}^{n}$ satisfying (1), let

$$
a_{G}(\mathbf{t}):=\sharp\{\sigma \in G / \mathbf{t}(\sigma)=\mathbf{t}\} .
$$

Then

$$
P_{G}\left(z_{1}, \ldots, z_{n}\right)=\frac{1}{\# G} \sum_{t \in S_{n}} a_{G}(\mathbf{t}) \mathbf{z}^{\mathrm{t}},
$$

where

$$
S_{n}:=\left\{\mathrm{t} \in \mathrm{N}_{0}^{n} / \sum_{i=1}^{n} i t_{i}=n\right\} .
$$

2. Simple Examples. For certain special series of groups, the Pólya polynomials are generally computable; cf. de Bruijn [2, Section 1]

(a) $G=\{e\} \Rightarrow P_{G}\left(z_{1}, \ldots, z_{n}\right)=z_{1}^{n}$ for any $n \in \mathbf{N}$,

(b) $G=\mathfrak{S}_{n}$.

Received August 31, 1979; revised May 2, 1980.

1980 Mathematics Subject Classification. Primary 12A55, 20 B25. 
For $t \in S_{n}$ (cf. (6)), we find with combinatorial considerations

$$
a_{\subseteq_{n}}(\mathbf{t})=\frac{n !}{\prod_{i=1}^{n} t_{i} ! i^{t_{i}}}
$$

and hence

$$
P_{S_{n}}\left(z_{1}, \ldots, z_{n}\right)=\sum_{t \in S_{n}}\left(\prod_{i=1}^{n} \frac{1}{t_{i} !}\left(\frac{z_{i}}{i}\right)^{t_{i}}\right)
$$

for instance

$$
\begin{array}{ll}
n=1: & P_{\Im_{1}}=z_{1}, \\
n=2: & P_{\Im_{2}}=\frac{1}{2}\left(z_{1}^{2}+z_{2}\right), \\
n=3: & P_{\Im_{3}}=\frac{1}{6}\left(z_{1}^{3}+3 z_{1} z_{2}+2 z_{3}\right), \\
n=4: & P_{\Im_{4}}=\frac{1}{24}\left(z_{1}^{4}+6 z_{1}^{2} z_{2}+8 z_{1} z_{3}+6 z_{4}+3 z_{2}^{2}\right) .
\end{array}
$$

(c) $G=\mathfrak{U}_{n}$.

For $\sigma \in \mathfrak{S}_{n}, \mathbf{t}:=\mathbf{t}(\sigma), m:=[n / 2]$, we have

$$
\begin{aligned}
\sigma \in \mathfrak{A}_{n} & \Leftrightarrow \operatorname{sign} \sigma=1 \Leftrightarrow(-1)^{t_{2}+t_{4}+\cdots+t_{2 m}}=1 \\
& \Leftrightarrow t_{2}+t_{4}+\cdots+t_{2 m} \in 2 \mathbf{N}_{0} .
\end{aligned}
$$

Furthermore,

$$
\bigwedge_{\sigma \in \mathfrak{A}_{n}} a_{\widetilde{\subseteq}_{n}}(\mathbf{t}(\sigma))=a_{\mathfrak{A}_{n}}(\mathbf{t}(\sigma))
$$

and therefore, with $\sharp \mathfrak{A}_{n}=\# S_{n} / 2$ and (7),

$$
P_{\mathfrak{A}_{n}}\left(z_{1}, \ldots, z_{n}\right)=2 \sum_{t \in A_{n}}\left(\prod_{i=1}^{n} \frac{1}{t_{i} !}\left(\frac{z_{i}}{i}\right)^{t_{i}}\right) \text {, }
$$

where

$$
A_{n}:=\left\{\mathrm{t} \in S_{n} / \sum_{i=1}^{m} t_{2_{i}} \in 2 \mathbf{N}_{0}\right\}
$$

Hence, we find the Pólya polynomial of the alternating group $\mathfrak{U}_{n}$ by taking the Pólya polynomial of the symmetric group $\widetilde{S}_{n}$, cancelling the terms with $t_{2}+t_{4}$ $+\cdots+t_{2 m} \in 2 \mathbf{N}_{0}$, and doubling all other coefficients. For instance:

$$
n=3: \quad P_{\mathfrak{A}_{3}}=\frac{1}{3}\left(z_{1}^{3}+2 z_{3}\right), \quad n=4: \quad P_{\mathfrak{A}_{4}}=\frac{1}{12}\left(z_{1}^{4}+8 z_{1} z_{3}+3 z_{2}^{2}\right) .
$$

3. Remarks on the Number-Theoretical Interpretation of Pólya polynomials. Any separable polynomial $f$ of degree $n$ over a ground field $k$ determines a Galois group $G=G_{f}$ as the permutation group of the roots of $f$. Let $\alpha_{1}, \ldots, \alpha_{n}$ be the roots of $f$.

$$
G_{f}\left(\left\{\begin{array}{l}
N:=k\left(\alpha_{1}, \ldots, \alpha_{n}\right) \\
K:=k\left(\alpha_{1}\right) \\
k
\end{array} \quad \mathscr{P}_{k} \text { will denote the set of all prime ideals in } k .\right.\right.
$$


Let $\mathfrak{p} \in \mathscr{P}_{k}$ be unramified in $K$ and have

$$
\begin{array}{cc}
t_{1} & \text { prime divisors in } K \text { of degree 1, } \\
t_{2} & \text { prime divisors in } K \text { of degree 2, } \\
: & \\
t_{n} & \text { prime divisors in } K \text { of degree } n .
\end{array}
$$

It is well known, that $\sum_{i=1}^{n} i t_{i}=n$.

$\mathbf{t}:=\left(t_{1}, \ldots, t_{n}\right)$ is called the type of $\mathfrak{p}$ in $K$, which is denoted by $\mathbf{t}=\mathbf{t}(\mathfrak{p})=$ $\mathbf{t}_{K \mid k}(\mathfrak{p})$.

Already Artin [1] ("Hilfssatz" in Abschnitt 2) and Hurwitz [4] ("Satz von Frobenius") proved that for $\mathfrak{p} \in \mathscr{P}_{k}$, which are unramified in $N$,

$$
\begin{aligned}
& \mathbf{t}_{N \mid k}(\mathfrak{p})=\left(t_{1}, \ldots, t_{n}\right) \Leftrightarrow\text { any } \sigma \in(N \mid k / \mathfrak{p}) \quad \text { (as a permutation of the roots of } f) \\
& \text { has the type }\left(t_{1}, \ldots, t_{n}\right): \\
& \bigwedge_{\sigma \in(N \mid k / p)} \mathbf{t}(\sigma)=\left(t_{1}, \ldots, t_{n}\right),
\end{aligned}
$$

where $(N \mid k / \mathfrak{p})$ denotes the Frobenius symbol.

Using Cebotarev's density theorem, we get

$$
\delta\left(\left\{\mathfrak{p} \in \mathscr{P}_{k} / \mathbf{t}(\mathfrak{p})=\left(t_{1}, \ldots, t_{n}\right)\right\}\right)=\frac{1}{\sharp G_{f}} a_{G_{f}}\left(t_{1}, \ldots, t_{n}\right),
$$

where $\delta$ denotes the Dirichlet-density (cf. (4)). But the right-hand side is a coefficient of the Pólya polynomial $P_{G_{f}}$ and can be determined purely grouptheoretically.

4. Results for Primitive Permutation Groups Up to Degree 20. I made use of an old version of the program system GROUP, developed by J. Neubüser, Aachen, and J. J. Cannon [3], to compute Pólya polynomials for given permutation groups. I coded and implemented a change in the algorithm determining all elements, so that, in principle, one is able to determine the Pólya polynomial of any given permutation group with the computer. The limitations are naturally memory-usage and time-consumption. Input for the program are generating elements, from which the program collects all occurring permutation types, and their frequencies, during the determination of all elements, so that the output can be the coefficients of the Pólya polynomial.

All primitive permutation groups up to degree 20 (determined by C. C. Sims [5]) were used as input to the program. The results (and thus the program) have been checked by hand for small group-orders and by comparison with theoretical results for $\mathfrak{A}_{n}, \mathfrak{S}_{n}$.

To identify the groups I used the notation of Sims [5]; for instance, 7.2 denotes the second group of degree 7 in Sims' list, and 11.6 the sixth group of degree 11 (which is the Mathieu group $M_{11}$ ).

The program only failed for the groups $16.19,16.20, \mathfrak{A}_{n}$ for $n=9,10, \ldots, 20$, $\Im_{n}$ for $n=8,9,10, \ldots, 20$, and for 12.4 , the Mathieu group $M_{12}$. All these groups have orders exceeding 40000 , for which the memory of the CDC 7600 is too small. But, since for $\mathfrak{A}_{n}, \Im_{n}$ we have theoretical results, only the groups $16.19,16.20$, and $12.4=M_{12}$ are still to be examined. 
Table 1 gives a list of all occurring permutation types and Table 2 gives the frequencies of their occurrence in the actual group (omitted types in Table 2 have frequency 0).

Examples. (a) To determine the Pólya-Polynomial of group $3.2=\mathbb{S}_{3}$ :

$$
\begin{array}{lc}
\text { occurring type } & \text { frequency }\left(=a_{3.2}\right. \text { (type), cf. (4)) } \\
a=(3,0,0) & 1 \\
b=(1,1,0) & 3 \\
c=(0,0,1) & \frac{2}{6}=\text { order of group }
\end{array}
$$

According to (5),

$$
\begin{aligned}
P_{\Im_{3}} & =\frac{1}{6}\left(1 \cdot\left(z_{1}, z_{2}, z_{3}\right)^{(3,0,0)}+3 \cdot\left(z_{1}, z_{2}, z_{3}\right)^{(1,1,0)}+2 \cdot\left(z_{1}, z_{2}, z_{3}\right)^{(0,0,1)}\right) \\
& =\frac{1}{(3)}\left(z_{1}^{3}+3 z_{1} z_{2}+2 z_{3}\right) .
\end{aligned}
$$

(b) Prime factorization in fields $k$ of degree 11 with group $11.6=M_{11}$ as Galois group of the Galois-hull:

occurring type

$$
\begin{array}{lr}
a=(11,0,0,0,0,0,0,0,0,0,0) & 1 \\
b=(3,4,0,0,0,0,0,0,0,0,0) & 165 \\
c=(3,0,0,2,0,0,0,0,0,0,0) & 990 \\
d=(2,0,3,0,0,0,0,0,0,0,0) & 440 \\
e=(1,1,0,0,0,0,0,1,0,0,0) & 1980 \\
f=(1,0,0,0,2,0,0,0,0,0,0) & 1584 \\
g=(0,1,1,0,0,1,0,0,0,0,0) & 1320 \\
h=(0,0,0,0,0,0,0,0,0,0,1) & \frac{1440}{7920}
\end{array}
$$

According to (8) this means: asymptotically we have to find

$$
\begin{array}{lrl}
\frac{1}{7920} & \text { of all prime ideals being } & \text { fully decomposed } \\
\frac{165}{7920}=\frac{1}{48} & \text { of all prime ideals having } & 3 \text { divisors of degree } 1 \\
\text { and } & 4 \text { divisors of degree } 2
\end{array}
$$




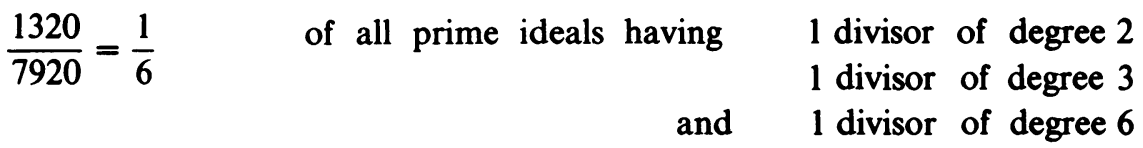

$\frac{1440}{7920}=\frac{2}{11} \quad$ of all prime ideals staying prime.

\section{TABLE 1}

Occurring permutation types of primitive permutation groups up to degree 20

Degree Permutation types

$$
3 \quad \begin{aligned}
a & =(3,0,0) \\
b & =(1,1,0) \\
c & =(0,0,1) \\
& a=(4,0,0,0) \\
b & =(2,1,0,0) \\
c & =(1,0,1,0) \\
d & =(0,2,0,0) \\
e & =(0,0,0,1)
\end{aligned}
$$

$5 \quad a=(5,0,0,0,0)$

$b=(3,1,0,0,0)$

$c=(2,0,1,0,0)$

$d=(1,2,0,0,0)$

$e=(1,0,0,1,0)$

$f=(0,1,1,0,0)$

$g=(0,0,0,0,1)$

$6 \quad a=(6,0,0,0,0,0)$

$b=(4,1,0,0,0,0)$

$c=(3,0,1,0,0,0)$

$d=(2,2,0,0,0,0)$

$e=(2,0,0,1,0,0)$

$f=(1,1,1,0,0,0)$

$g=(1,0,0,0,1,0)$

$h=(0,3,0,0,0,0)$

$i=(0,1,0,1,0,0)$

$k=(0,0,2,0,0,0)$

$l=(0,0,0,0,0,1)$

$7 \quad a=(7,0,0,0,0,0,0)$

$b=(5,1,0,0,0,0,0)$

$c=(4,0,1,0,0,0,0)$

$d=(3,2,0,0,0,0,0)$

$e=(3,0,0,1,0,0,0)$

$f=(2,1,1,0,0,0,0)$

$g=(2,0,0,0,1,0,0)$ 
Degree Permutation types

$h=(1,3,0,0,0,0,0)$

$i=(1,1,0,1,0,0,0)$

$k=(1,0,2,0,0,0,0)$

$l=(1,0,0,0,0,1,0)$

$m=(0,2,1,0,0,0,0)$

$n=(0,1,0,0,1,0,0)$

$o=(0,0,1,1,0,0,0)$

$p=(0,0,0,0,0,0,1)$

8

$a=(8,0,0,0,0,0,0,0)$

$b=(5,0,1,0,0,0,0,0)$

$c=(4,2,0,0,0,0,0,0)$

$d=(3,0,0,0,1,0,0,0)$

$e=(2,3,0,0,0,0,0,0)$

$f=(2,1,0,1,0,0,0,0)$

$g=(2,0,2,0,0,0,0,0)$

$h=(2,0,0,0,0,1,0,0)$

$i=(1,2,1,0,0,0,0,0)$

$k=(1,0,0,0,0,0,1,0)$

$l=(0,4,0,0,0,0,0,0)$

$m=(0,1,0,0,0,1,0,0)$

$n=(0,0,1,0,1,0,0,0)$

$o=(0,0,0,2,0,0,0,0)$

$p=(0,0,0,0,0,0,0,1)$

$9 \quad a=(9,0,0,0,0,0,0,0,0)$

$b=(3,3,0,0,0,0,0,0,0)$

$c=(3,0,2,0,0,0,0,0,0)$

$d=(2,0,0,0,0,0,1,0,0)$

$e=(1,4,0,0,0,0,0,0,0)$

$f=(1,1,0,0,0,1,0,0,0)$

$g=(1,0,0,2,0,0,0,0,0)$

$h=(1,0,0,0,0,0,0,1,0)$

$i=(0,0,3,0,0,0,0,0,0)$

$k=(0,0,1,0,0,1,0,0,0)$

$l=(0,0,0,0,0,0,0,0,1)$

10

$a=(10,0,0,0,0,0,0,0,0,0)$

$b=(4,3,0,0,0,0,0,0,0,0)$

$c=(2,4,0,0,0,0,0,0,0,0)$

$d=(2,0,0,2,0,0,0,0,0,0)$

$e=(2,0,0,0,0,0,0,1,0,0)$

$f=(1,0,3,0,0,0,0,0,0,0)$

$g=(1,0,1,0,0,1,0,0,0,0)$

$h=(0,5,0,0,0,0,0,0,0,0)$

$i=(0,1,0,2,0,0,0,0,0,0)$

$k=(0,1,0,0,0,0,0,1,0,0)$ 
Degree Permutation types

$$
\begin{aligned}
l & =(0,0,0,0,2,0,0,0,0,0) \\
m & =(0,0,0,0,0,0,0,0,0,1)
\end{aligned}
$$

11

$$
\begin{aligned}
a & =(11,0,0,0,0,0,0,0,0,0,0) \\
b & =(3,4,0,0,0,0,0,0,0,0,0) \\
c & =(3,0,0,2,0,0,0,0,0,0,0) \\
d & =(2,0,3,0,0,0,0,0,0,0,0) \\
e & =(1,5,0,0,0,0,0,0,0,0,0) \\
f & =(1,1,0,0,0,0,0,1,0,0,0) \\
g & =(1,0,0,0,2,0,0,0,0,0,0) \\
h & =(1,0,0,0,0,0,0,0,0,1,0) \\
i & =(0,1,1,0,0,1,0,0,0,0,0) \\
k & =(0,0,0,0,0,0,0,0,0,0,1)
\end{aligned}
$$$$
a=(12,0,0,0,0,0,0,0,0,0,0,0)
$$$$
b=(4,4,0,0,0,0,0,0,0,0,0,0)
$$$$
c=(3,0,3,0,0,0,0,0,0,0,0,0)
$$$$
d=(2,5,0,0,0,0,0,0,0,0,0,0)
$$$$
e=(2,0,0,0,2,0,0,0,0,0,0,0)
$$$$
f=(2,0,0,0,0,0,0,0,0,1,0,0)
$$$$
g=(1,1,1,0,0,1,0,0,0,0,0,0)
$$$$
h=(1,0,0,0,0,0,0,0,0,0,1,0)
$$$$
i=(0,6,0,0,0,0,0,0,0,0,0,0)
$$$$
k=(0,2,0,2,0,0,0,0,0,0,0,0)
$$$$
l=(0,0,4,0,0,0,0,0,0,0,0,0)
$$$$
m=(0,0,0,3,0,0,0,0,0,0,0,0)
$$$$
n=(0,0,0,1,0,0,0,1,0,0,0,0)
$$$$
o=(0,0,0,0,0,2,0,0,0,0,0,0)
$$$$
p=(0,0,0,0,0,0,0,0,0,0,0,1)
$$

$$
\begin{aligned}
a & =(13,0,0,0,0,0,0,0,0,0,0,0,0) \\
b & =(5,4,0,0,0,0,0,0,0,0,0,0,0) \\
c & =(4,0,3,0,0,0,0,0,0,0,0,0,0) \\
d & =(2,1,1,0,0,1,0,0,0,0,0,0,0) \\
e & =(1,6,0,0,0,0,0,0,0,0,0,0,0) \\
f & =(1,2,0,2,0,0,0,0,0,0,0,0,0) \\
g & =(1,0,4,0,0,0,0,0,0,0,0,0,0) \\
h & =(1,0,0,3,0,0,0,0,0,0,0,0,0) \\
i & =(1,0,0,1,0,0,0,1,0,0,0,0,0) \\
k & =(1,0,0,0,0,2,0,0,0,0,0,0,0) \\
l & =(1,0,0,0,0,0,0,0,0,0,0,1,0) \\
m & =(0,0,0,0,0,0,0,0,0,0,0,0,1)
\end{aligned}
$$

$$
\begin{aligned}
& a=(14,0,0,0,0,0,0,0,0,0,0,0,0,0) \\
& b=(2,6,0,0,0,0,0,0,0,0,0,0,0,0) \\
& c=(2,0,4,0,0,0,0,0,0,0,0,0,0,0) \\
& d=(2,0,0,3,0,0,0,0,0,0,0,0,0,0)
\end{aligned}
$$


Degree Permutation types

$e=(2,0,0,0,0,2,0,0,0,0,0,0,0,0)$

$f=(2,0,0,0,0,0,0,0,0,0,0,1,0,0)$

$g=(1,0,0,0,0,0,0,0,0,0,0,0,1,0)$

$h=(0,7,0,0,0,0,0,0,0,0,0,0,0,0)$

$i=(0,0,0,0,0,0,2,0,0,0,0,0,0,0)$

$k=(0,0,0,0,0,0,0,0,0,0,0,0,0,1)$

$a=(15,0,0,0,0,0,0,0,0,0,0,0,0,0,0)$

$b=(7,4,0,0,0,0,0,0,0,0,0,0,0,0,0)$

$c=(3,6,0,0,0,0,0,0,0,0,0,0,0,0,0)$

$d=(3,2,0,2,0,0,0,0,0,0,0,0,0,0,0)$

$e=(3,0,4,0,0,0,0,0,0,0,0,0,0,0,0)$

$f=(1,1,2,0,0,1,0,0,0,0,0,0,0,0,0)$

$g=(1,1,0,3,0,0,0,0,0,0,0,0,0,0,0)$

$h=(1,0,0,0,0,0,2,0,0,0,0,0,0,0,0)$

$i=(0,0,5,0,0,0,0,0,0,0,0,0,0,0,0)$

$k=(0,0,1,0,0,2,0,0,0,0,0,0,0,0,0)$

$l=(0,0,0,0,3,0,0,0,0,0,0,0,0,0,0)$

$m=(0,0,0,0,0,0,0,0,0,0,0,0,0,0,1)$

16

$a=(16,0,0,0,0,0,0,0,0,0,0,0,0,0,0,0)$

$b=(8,4,0,0,0,0,0,0,0,0,0,0,0,0,0,0)$

$c=(4,6,0,0,0,0,0,0,0,0,0,0,0,0,0,0)$

$d=(4,0,4,0,0,0,0,0,0,0,0,0,0,0,0,0)$

$e=(2,1,2,0,0,1,0,0,0,0,0,0,0,0,0,0)$

$f=(2,1,0,3,0,0,0,0,0,0,0,0,0,0,0,0)$

$g=(1,0,5,0,0,0,0,0,0,0,0,0,0,0,0,0)$

$h=(1,0,1,0,0,2,0,0,0,0,0,0,0,0,0,0)$

$i=(1,0,0,0,3,0,0,0,0,0,0,0,0,0,0,0)$

$k=(1,0,0,0,0,0,0,0,0,0,0,0,0,0,1,0)$

$l=(0,8,0,0,0,0,0,0,0,0,0,0,0,0,0,0)$

$m=(0,2,0,0,0,2,0,0,0,0,0,0,0,0,0,0)$

$n=(0,0,0,4,0,0,0,0,0,0,0,0,0,0,0,0)$

$o=(0,0,0,1,0,0,0,0,0,0,0,1,0,0,0,0)$

$p=(0,0,0,0,0,0,0,2,0,0,0,0,0,0,0,0)$

$17 \quad a=(17,0,0,0,0,0,0,0,0,0,0,0,0,0,0,0,0)$

$b=(5,6,0,0,0,0,0,0,0,0,0,0,0,0,0,0,0)$

$c=(3,1,0,3,0,0,0,0,0,0,0,0,0,0,0,0,0)$

$d=(2,0,5,0,0,0,0,0,0,0,0,0,0,0,0,0,0)$

$e=(2,0,1,0,0,2,0,0,0,0,0,0,0,0,0,0,0)$

$f=(2,0,0,0,3,0,0,0,0,0,0,0,0,0,0,0,0)$

$g=(2,0,0,0,0,0,0,0,0,0,0,0,0,0,1,0,0)$

$h=(1,8,0,0,0,0,0,0,0,0,0,0,0,0,0,0,0)$

$i=(1,0,0,4,0,0,0,0,0,0,0,0,0,0,0,0,0)$

$k=(1,0,0,0,0,0,0,2,0,0,0,0,0,0,0,0,0)$ 
Degree Permutation types

$$
\begin{aligned}
l & =(1,0,0,0,0,0,0,0,0,0,0,0,0,0,0,1,0) \\
m & =(0,1,1,0,0,0,0,0,0,0,0,1,0,0,0,0,0) \\
n & =(0,1,0,0,1,0,0,0,0,1,0,0,0,0,0,0,0) \\
o & =(0,0,0,0,0,0,0,0,0,0,0,0,0,0,0,0,1)
\end{aligned}
$$

$a=(18,0,0,0,0,0,0,0,0,0,0,0,0,0,0,0,0,0)$

$b=(2,8,0,0,0,0,0,0,0,0,0,0,0,0,0,0,0,0)$

$c=(2,0,0,0,4,0,0,0,0,0,0,0,0,0,0,0,0,0)$

$d=(2,0,0,0,0,0,0,2,0,0,0,0,0,0,0,0,0,0)$

$e=(2,0,0,0,0,0,0,0,0,0,0,0,0,0,0,1,0,0)$

$f=(1,0,0,0,0,0,0,0,0,0,0,0,0,0,0,0,1,0)$

$g=(0,9,0,0,0,0,0,0,0,0,0,0,0,0,0,0,0,0)$

$h=(0,0,6,0,0,0,0,0,0,0,0,0,0,0,0,0,0,0)$

$i=(0,0,0,0,0,3,0,0,0,0,0,0,0,0,0,0,0,0)$

$k=(0,0,0,0,0,0,0,0,2,0,0,0,0,0,0,0,0,0)$

$l=(0,0,0,0,0,0,0,0,0,0,0,0,0,0,0,0,0,1)$

$a=(19,0,0,0,0,0,0,0,0,0,0,0,0,0,0,0,0,0,0)$

$b=(1,9,0,0,0,0,0,0,0,0,0,0,0,0,0,0,0,0,0)$

$c=(1,0,6,0,0,0,0,0,0,0,0,0,0,0,0,0,0,0,0)$

$d=(1,0,0,0,0,3,0,0,0,0,0,0,0,0,0,0,0,0,0)$

$e=(1,0,0,0,0,0,0,0,2,0,0,0,0,0,0,0,0,0,0)$

$f=(1,0,0,0,0,0,0,0,0,0,0,0,0,0,0,0,0,1,0)$

$g=(0,0,0,0,0,0,0,0,0,0,0,0,0,0,0,0,0,0,1)$

$a=(20,0,0,0,0,0,0,0,0,0,0,0,0,0,0,0,0,0,0,0)$

$b=(2,9,0,0,0,0,0,0,0,0,0,0,0,0,0,0,0,0,0,0)$

$c=(2,0,6,0,0,0,0,0,0,0,0,0,0,0,0,0,0,0,0,0)$

$d=(2,0,0,0,0,3,0,0,0,0,0,0,0,0,0,0,0,0,0,0)$

$e=(2,0,0,0,0,0,0,0,2,0,0,0,0,0,0,0,0,0,0,0)$

$f=(2,0,0,0,0,0,0,0,0,0,0,0,0,0,0,0,0,1,0,0)$

$g=(1,0,0,0,0,0,0,0,0,0,0,0,0,0,0,0,0,0,1,0)$

$h=(0,10,0,0,0,0,0,0,0,0,0,0,0,0,0,0,0,0,0,0)$

$i=(0,0,0,5,0,0,0,0,0,0,0,0,0,0,0,0,0,0,0,0)$

$k=(0,0,0,0,4,0,0,0,0,0,0,0,0,0,0,0,0,0,0,0)$

$l=(0,0,0,0,0,0,0,0,0,2,0,0,0,0,0,0,0,0,0,0)$

$m=(0,0,0,0,0,0,0,0,0,0,0,0,0,0,0,0,0,0,0,1)$

TABLE 2

Frequencies of permutation types in primitive permutation groups up to degree 20

$\begin{array}{ccrl}\text { Degree } & \text { Group } & \text { Order } & \text { Occurring frequencies } \\ 3 & 3.2 & 6 & 1 a, 3 b, 2 c \\ 4 & 4.1 & 12 & 1 a, 8 c, 3 d \\ & 4.2 & 24 & a, 6 b, 8 c, 3 d, 6 e\end{array}$




\begin{tabular}{|c|c|c|c|}
\hline Degree & Group & Order & Occurring frequencies \\
\hline \multirow[t]{5}{*}{5} & 5.1 & 5 & $1 a, 4 g$ \\
\hline & 5.2 & 10 & $1 a, 5 d, 4 g$ \\
\hline & 5.3 & 20 & $1 a, 5 d, 10 e, 4 g$ \\
\hline & 5.4 & 60 & $1 a, 20 c, 15 d, 24 g$ \\
\hline & 5.5 & 120 & $1 a, 10 b, 20 c, 15 d, 30 e, 20 f, 24 g$ \\
\hline \multirow[t]{4}{*}{6} & 6.1 & 60 & $1 a, 15 d, 24 g, 20 k$ \\
\hline & 6.2 & 120 & $1 a, 15 d, 30 e, 24 g, 10 h, 20 k, 20 l$ \\
\hline & 6.3 & 360 & $1 a, 40 c, 45 d, 144 g, 90 i, 40 k$ \\
\hline & 6.4 & 720 & $\begin{array}{l}1 a, 15 b, 40 c, 45 d, 90 e, 120 f, 144 g, 15 h \\
90 i, 40 k, 120 l\end{array}$ \\
\hline \multirow[t]{7}{*}{7} & 7.1 & 7 & $1 a, 6 p$ \\
\hline & 7.2 & 14 & $1 a, 7 h, 6 p$ \\
\hline & 7.3 & 21 & $1 a, 14 k, 6 p$ \\
\hline & 7.4 & 42 & $1 a, 7 h, 14 k, 14 l, 6 p$ \\
\hline & 7.5 & 168 & $1 a, 21 d, 56 k, 42 i, 48 p$ \\
\hline & 7.6 & 2520 & $1 a, 70 c, 105 d, 504 g, 630 i, 280 k, 210 m, 720 p$ \\
\hline & 7.7 & 5040 & $\begin{array}{l}1 a, 21 b, 70 c, 105 d, 210 e, 420 f, 504 g, 105 h \\
630 i, 280 k, 840 l, 210 m, 504 n, 420 o, 720 p\end{array}$ \\
\hline \multirow[t]{6}{*}{8} & 8.1 & 56 & $1 a, 48 k, 7 l$ \\
\hline & 8.2 & 168 & $1 a, 56 g, 48 k, 7 l, 56 m$ \\
\hline & 8.3 & 168 & $1 a, 56 g, 48 k, 21 l, 42 o$ \\
\hline & 8.4 & 336 & $1 a, 28 e, 56 g, 56 h, 48 k, 21 l, 42 o, 84 p$ \\
\hline & 8.5 & 1344 & $1 a, 42 c, 168 f, 224 g, 384 k, 49 l, 224 m, 252 o$ \\
\hline & 8.6 & 20160 & $\begin{array}{l}1 a, 112 b, 210 c, 1344 d, 2520 f, 1120 \mathrm{~g}, 1680 i \\
5760 k, 105 l, 3360 \mathrm{~m}, 2688 n, 1260 o\end{array}$ \\
\hline \multirow[t]{9}{*}{9} & 9.1 & 36 & $1 a, 9 e, 18 g, 8 i$ \\
\hline & 9.2 & 72 & $1 a, 12 b, 9 e, 18 g, 8 i, 24 k$ \\
\hline & 9.3 & 72 & $1 a, 9 e, 18 g, 36 h, 8 i$ \\
\hline & 9.4 & 72 & $1 a, 9 e, 54 \mathrm{~g}, 8 i$ \\
\hline & 9.5 & 144 & $1 a, 12 b, 9 e, 54 g, 36 h, 8 i, 24 k$ \\
\hline & 9.6 & 216 & $1 a, 24 c, 9 e, 72 f, 54 g, 56 i$ \\
\hline & 9.7 & 432 & $1 a, 36 b, 24 c, 9 e, 72 f, 54 g, 108 h, 56 i, 72 k$ \\
\hline & 9.8 & 504 & $1 a, 216 d, 63 e, 56 i, 168 l$ \\
\hline & 9.9 & 1512 & $1 a, 168 c, 216 d, 63 e, 504 f, 56 i, 504 l$ \\
\hline \multirow[t]{7}{*}{10} & 10.1 & 60 & $1 a, 15 c, 20 f, 24 l$ \\
\hline & 10.2 & 120 & $1 a, 10 b, 15 c, 20 f, 20 g, 30 i, 24 l$ \\
\hline & 10.3 & 360 & $1 a, 45 c, 90 d, 80 f, 144 l$ \\
\hline & 10.4 & 720 & $1 a, 30 b, 45 c, 90 d, 80 f, 240 g, 90 i, 144 l$ \\
\hline & 10.5 & 720 & $1 a, 45 c, 90 d, 180 e, 80 f, 36 h, 144 l, 144 m$ \\
\hline & 10.6 & 720 & $1 a, 45 c, 270 d, 80 f, 180 k, 144 l$ \\
\hline & 10.7 & 1440 & $\begin{array}{l}1 a, 30 b, 45 c, 270 d, 180 e, 80 f, 240 g, 36 h \\
90 i, 180 k, 144 l, 144 m\end{array}$ \\
\hline
\end{tabular}




\begin{tabular}{|c|c|c|c|}
\hline Degree & e Group & Order & Occurring frequencies \\
\hline 11 & 11.1 & 11 & $1 a, 10 k$ \\
\hline & 11.2 & 22 & $1 a, 11 e, 10 k$ \\
\hline & 11.3 & 55 & $1 a, 44 g, 10 k$ \\
\hline & 11.4 & 110 & $1 a, 11 e, 44 g, 44 h, 10 k$ \\
\hline & 11.5 & 660 & $1 a, 55 b, 110 d, 264 g, 110 i, 120 k$ \\
\hline & 11.6 & 7920 & $1 a, 165 b, 990 c, 440 d, 1980 f, 1584 g, 1320 i, 1440 k$ \\
\hline 12 & 12.1 & 660 & $1 a, 264 e, 120 h, 55 i, 110 l, 110 o$ \\
\hline & 12.2 & 1320 & $\begin{array}{l}1 a, 66 d, 264 e, 264 f, 120 h, 55 i, 110 l, 110 m \\
110 o, 220 p\end{array}$ \\
\hline & 12.3 & 7920 & $1 a, 165 b, 440 c, 1584 e, 1320 g, 1440 h, 990 k, 1980 n$ \\
\hline 13 & 13.1 & 13 & $1 a, 12 m$ \\
\hline & 13.2 & 26 & $1 a, 13 e, 12 m$ \\
\hline & 13.3 & 39 & $1 a, 26 g, 12 m$ \\
\hline & 13.4 & 52 & $1 a, 13 e, 26 h, 12 m$ \\
\hline & 13.5 & 78 & $1 a, 13 e, 26 g, 26 k, 12 m$ \\
\hline & 13.6 & 156 & $1 a, 13 e, 26 g, 26 h, 26 k, 52 l, 12 m$ \\
\hline & 13.7 & 5616 & $1 a, 117 b, 104 c, 936 d, 702 f, 624 g, 1404 i, 1728 m$ \\
\hline 14 & 14.1 & 1092 & $1 a, 91 b, 182 c, 182 e, 168 g, 468 i$ \\
\hline & 14.2 & 2184 & $\begin{array}{l}1 a, 91 b, 182 c, 182 d, 182 e, 364 f, 168 g, 78 h, \\
468 i, 468 k\end{array}$ \\
\hline 15 & 15.1 & 360 & $1 a, 45 c, 40 e, 90 \mathrm{~g}, 40 i, 144 l$ \\
\hline & 15.2 & 720 & $1 a, 15 b, 60 c, 40 e, 120 f, 180 g, 40 i, 120 k, 144 l$ \\
\hline & 15.3 & 2520 & $1 a, 105 c, 280 e, 630 g, 720 h, 70 i, 210 k, 504 l$ \\
\hline & 15.4 & 20160 & $\begin{array}{l}1 a, 105 b, 210 c, 1260 d, 1120 e, 3360 f, 2520 g \\
5760 h, 112 i, 1680 k, 1344 l, 2688 m\end{array}$ \\
\hline 16 & 16.1 & 80 & $1 a, 64 i, 15 l$ \\
\hline & 16.2 & 160 & $1 a, 20 c, 64 i, 15 l, 60 n$ \\
\hline & 16.3 & 240 & $1 a, 32 g, 64 i, 128 k, 15 l$ \\
\hline & 16.4 & 288 & $1 a, 12 c, 16 d, 64 g, 96 h, 15 l, 48 m, 36 n$ \\
\hline & 16.5 & 320 & $1 a, 20 c, 80 f, 64 i, 15 l, 60 n, 80 p$ \\
\hline & 16.6 & 480 & $1 a, 20 c, 32 g, 160 h, 64 i, 128 k, 15 l, 60 n$ \\
\hline & 16.7 & 576 & $1 a, 36 c, 16 d, 144 f, 64 g, 15 l, 48 m, 108 n, 144 p$ \\
\hline & 16.8 & 576 & $1 a, 60 c, 16 d, 64 g, 192 h, 15 l, 48 m, 180 n$ \\
\hline & 16.9 & 960 & $1 a, 60 c, 80 d, 384 i, 15 l, 240 m, 180 n$ \\
\hline & 16.10 & 960 & $1 a, 20 c, 240 f, 32 g, 160 h, 64 i, 128 k, 15 l, 60 n, 240 p$ \\
\hline & 16.11 & 960 & $1 a, 60 c, 320 g, 384 i, 15 l, 180 n$ \\
\hline & 16.12 & 1152 & $\begin{array}{l}1 a, 12 b, 60 c, 16 d, 96 e, 144 f, 64 g, 192 h, 51 l \\
48 m, 228 n, 96 o, 144 p\end{array}$ \\
\hline & 16.13 & 1920 & $\begin{array}{l}1 a, 20 b, 60 c, 80 d, 160 e, 240 f, 384 i, 75 l, 240 m \\
260 n, 160 o, 240 p\end{array}$ \\
\hline & 16.14 & 1920 & $1 a, 100 c, 240 f, 320 g, 320 h, 384 i, 15 l, 300 n, 240 p$ \\
\hline & 16.15 & 2880 & $\begin{array}{l}1 a, 60 c, 160 d, 352 g, 480 h, 384 i, 768 k, 15 l \\
480 m, 180 n\end{array}$ \\
\hline
\end{tabular}




\begin{tabular}{|c|c|c|c|}
\hline Degree & Group & Order & Occurring frequencies \\
\hline & 16.16 & 5760 & $\begin{array}{l}1 a, 180 c, 160 d, 720 f, 352 g, 1440 h, 384 i, 768 k \\
15 l, 480 m, 540 n, 720 p\end{array}$ \\
\hline & 16.17 & 5760 & $\begin{array}{l}1 a, 180 c, 160 d, 720 f, 640 g, 2304 i, 15 l, 480 m \\
540 n, 720 p\end{array}$ \\
\hline & 16.18 & 11520 & $\begin{array}{l}1 a, 30 b, 240 c, 160 d, 960 e, 1440 f, 640 g, 1920 h \\
2304 i, 105 l, 480 m, 840 n, 960 o, 1440 p\end{array}$ \\
\hline 17 & 17.1 & 17 & $1 a, 16 o$ \\
\hline & 17.2 & 34 & $1 a, 17 h, 16 o$ \\
\hline & 17.3 & 68 & $1 a, 17 h, 34 i, 16 o$ \\
\hline & 17.4 & 136 & $1 a, 17 h, 34 i, 68 k, 16 o$ \\
\hline & 17.5 & 272 & $1 a, 17 h, 34 i, 68 k, 136 l, 16 o$ \\
\hline & 17.6 & 4080 & $1 a, 272 d, 544 f, 1088 g, 255 h, 1920 o$ \\
\hline & 17.7 & 8160 & $\begin{array}{l}1 a, 68 b, 272 d, 1360 e, 544 f, 1088 g, 255 h, 1020 i \\
1632 n, 1920 o\end{array}$ \\
\hline & 17.8 & 16320 & $\begin{array}{l}1 a, 68 b, 1360 c, 272 d, 1360 e, 544 f, 1088 g, 255 h \\
1020 i, 4080 k, 2720 m, 1632 n, 1920 o\end{array}$ \\
\hline 18 & 18.1 & 2448 & $1 a, 153 b, 306 c, 612 d, 288 f, 272 h, 816 k$ \\
\hline & 18.2 & 4896 & $\begin{array}{l}1 a, 153 b, 306 c, 612 d, 1224 e, 288 f, 136 g, 272 h \\
272 i, 816 k, 816 l\end{array}$ \\
\hline 19 & 19.1 & 19 & $1 a, 18 g$ \\
\hline & 19.2 & 38 & $1 a, 19 b, 18 g$ \\
\hline & 19.3 & 57 & $1 a, 38 c, 18 g$ \\
\hline & 19.4 & 114 & $1 a, 19 b, 38 c, 38 d, 18 g$ \\
\hline & 19.5 & 171 & $1 a, 38 c, 114 e, 18 g$ \\
\hline & 19.6 & 342 & $1 a, 19 b, 38 c, 38 d, 114 e, 114 f, 18 g$ \\
\hline 20 & 20.1 & 3420 & $1 a, 380 c, 1140 e, 360 \mathrm{~g}, 171 h, 684 k, 684 l$ \\
\hline & 20.2 & 6840 & $\begin{array}{l}1 a, 190 b, 380 c, 380 d, 1140 e, 1140 f, 360 g, 171 h \\
342 i, 684 k, 684 l, 1368 m\end{array}$ \\
\hline
\end{tabular}

Mathematisches Institut der Universität zu Köln

Weyertal 86-90

$5000 \mathrm{Köln} \mathrm{41,} \mathrm{West} \mathrm{Germany}$

1. E. Artin, “Über die Zeta-Funktion gewisser algebraischer Zahlkörper,” Math. Ann., v. 89, 1923, pp. 147-156.

2. N. G. DE BruUN, "Pólya's Abzähl-Theorie: Muster für Graphen und chemische Verbindungen," Selecta Mathematica III (Heidelberger Taschenbücher), v. 86, Springer-Verlag, Heidelberg, 1971, pp. $1-26$.

3. J. J. Cannon, A simple language for running group jobs, Tech. Rep., Dept. Pure Math., Univ. of Sydney, 1975.

4. A. HuRwitz, "Über Beziehungen zwischen den Primidealen eines algebraischen Körpers und den Substitutionen seiner Gruppe," Math. Z., v. 25, 1926, pp. 661-665.

5. C. C. Sms, "Computational methods in the study of permutation groups," Computational Problems in Abstract Algebra (Proc. Conf., Oxford, 1967), Pergamon Press, Oxford, 1970, pp. 169-183. 